\title{
Estimating Tessellation Parameter Intervals for Rational Curves and Surfaces
}

Thomas W. Sederberg

tom@cs.byu.edu

Jianmin Zheng

Follow this and additional works at: https://scholarsarchive.byu.edu/facpub

Part of the Computer Sciences Commons

\section{Original Publication Citation}

J. Zheng and T. W. Sederberg, "Estimating tessellation parameter intervals for rational curves and surfaces," ACM Transactions on Graphics, 19, 1, 56-77, 2.

\section{BYU ScholarsArchive Citation}

Sederberg, Thomas W. and Zheng, Jianmin, "Estimating Tessellation Parameter Intervals for Rational Curves and Surfaces" (2000). Faculty Publications. 1111.

https://scholarsarchive.byu.edu/facpub/1111

This Peer-Reviewed Article is brought to you for free and open access by BYU ScholarsArchive. It has been accepted for inclusion in Faculty Publications by an authorized administrator of BYU ScholarsArchive. For more information, please contact ellen_amatangelo@byu.edu. 


\title{
Estimating Tessellation Parameter Intervals for Rational Curves and Surfaces
}

\author{
Jianmin Zheng \\ Department of Applied Mathematics \\ Zhejiang University \\ Hangzhou, 310027, P.R.CHINA \\ and \\ Thomas W. Sederberg \\ Department of Computer Science \\ Brigham Young University \\ Provo, Utah 84602
}

\begin{abstract}
This paper presents a method for determining a priori a constant parameter interval with which a rational curve or surface can be tessellated such that the deviation of the curve or surface from its piecewise linear approximation is within a specified tolerance. The parameter interval is estimated based on information about the second order derivatives in the homogeneous coordinates, instead of using affine coordinates directly. This new step size can be found with roughly the same amount of computation as the step size presented in [Cheng 1992], though it can be proven to always be larger than Cheng's step size. In fact, numerical experiments show the new step is typically orders of magnitude larger than the step size in [Cheng 1992]. Furthermore, for rational cubic and quartic curves, the new step size is generally twice as large as the step size found by computing bounds on the Bernstein polynomial coefficients of the second derivatives function.
\end{abstract}

Categories and Subject Descriptors: I.3.5 [Computer Graphics]: Computational Geometry and Object Modelling-geometric algorithms; J.6 [Computer Applications]: Computer-Aided Engineering-computer-aided design

General Terms: Algorithms

Additional Key Words and Phrases: Rational curves and surfaces, tessellation, flatness, derivative bounds, step size, projection distance

\section{INTRODUCTION}

In computer graphics and modeling, parametric curves and surfaces are often tessellated into piecewise linear segments for rendering [Lane and Carpenter 1979; Rappoport 1991; Abi-Ezzi and Wozny 1991; Klassen 1994] mesh generation [Sheng and Hirsch 1992; Piegl and Richard 1995] and surface/surface intersection [Wang 1984; Filip et al. 1986]. In such applications, the approximation error is typically taken as the maximal distance between the original and the approximating segments.

Many criteria and methods have been developed for the task of assuring that a piecewise linear tessellation satisfies an error bound [Lane and Riesenfeld 1980; Schaback 1993; Elber 1996; Tookey and Cripps 1997]. One popular approach is to determine a global parameter interval or step size $\delta$ that is valid over the entire domain

Name: Jianmin Zheng

Affiliation: Zhejiang University

Address: Hangzhou, 310027, P.R.CHINA

Name: Thomas W. Sederberg

Affiliation: Brigham Young University

Address: Provo, Utah 84602; Email: tom@byu.edu

Permission to make digital or hard copies of part or all of this work for personal or classroom use is granted without fee provided that copies are not made or distributed for profit or direct commercial advantage and that copies show this notice on the first page or initial screen of a display along with the full citation. Copyrights for components of this work owned by others than ACM must be honored. Abstracting with credit is permitted. To copy otherwise, to republish, to post on servers, to redistribute to lists, or to use any component of this work in other works, requires prior specific permission and/or a fee. Permissions may be requested from Publications Dept, ACM Inc., 1515 Broadway, New York, NY 10036 USA, fax +1 (212) 869-0481, or permissions@acm.org. 
[Wang 1984; Filip et al. 1986; Cheng 1992; Sheng and Hirsch 1992; Klassen 1994]. Then the tessellation can be generated either by recursively splitting the curve or surface in half to a depth computed from $\delta$, or by just sampling the curve or surface at points separated by a parameter interval $\delta$. Such an approach lends itself well to forward differencing. A step size should ideally be as large as possible without violating the prescribed tolerance. The calculation of such a step size is usually not easy for rational curves and surfaces.

The general approach to obtaining such a step size is to establish a relatively simple relationship between the step size and a bound on the maximal deviation. Once the bound is found, the step size follows immediately. Based on theorems from approximation theory [de Boor 1978], Wang proposed using bounds on second order derivatives of the curve or surface to estimate deviation from the linear approximation [Wang 1984]. This approach was later enhanced by Filip et al [Filip et al. 1986]. For a $C^{2}$ continuous parametric curve $\mathbf{r}(t), t \in[\alpha, \beta]$, a bound on the displacement of $\mathbf{r}(t)$ from its linear approximation $\mathbf{L}(t)$ which interpolates $\mathbf{r}(\alpha)$ and $\mathbf{r}(\beta)$ is given by

$$
\sup _{t \in[\alpha, \beta]}\|\mathbf{r}(t)-\mathbf{L}(t)\| \leq \frac{1}{8} \delta^{2} \sup _{t \in[\alpha, \beta]}\left\|\mathbf{r}^{\prime \prime}(t)\right\|, \quad \delta=\beta-\alpha
$$

For a $C^{2}$ continuous parametric surface $\mathbf{r}(u, v)$ defined over a right triangle $T$ with two vertical edge lengths of $\delta_{u}$ and $\delta_{v}$, a bound on the deviation of $\mathbf{r}(u, v)$ from the base triangle $\mathbf{L}(u, v)$ which linearly interpolates $\mathbf{r}(u, v)$ at three vertices of $T$ is

$$
\sup _{(u, v) \in T}\|\mathbf{r}(u, v)-\mathbf{L}(u, v)\| \leq \frac{1}{8}\left(D_{u u} \delta_{u}^{2}+2 D_{u v} \delta_{u} \delta_{v}+D_{v v} \delta_{v}^{2}\right)
$$

where

$$
D_{u u}=\sup _{(u, v) \in T}\left\|\frac{\partial^{2} \mathbf{r}(u, v)}{\partial u^{2}}\right\|, D_{u v}=\sup _{(u, v) \in T}\left\|\frac{\partial^{2} \mathbf{r}(u, v)}{\partial u \partial v}\right\|, D_{v v}=\sup _{(u, v) \in T}\left\|\frac{\partial^{2} \mathbf{r}(u, v)}{\partial v^{2}}\right\|
$$

In these formulas, we need to compute the sup's of the second order (partial) derivatives over the domain of the curve or surface. For polynomial curves and surfaces, the convex hull property of the control points of the Bezier representation offers a straightforward way to compute upper bounds. For rational curves and surfaces, estimating the sup's of their second order (partial) derivatives is much more expensive. The rational case is important not only because it is more powerful in modeling than the polynomial scheme and because NURBS have become an industry standard in CAD/CAM systems, but also because perspective transformation changes a polynomial curve or surface to a rational curve or surface. (While these formulae apply to any $C^{2}$ curve or surface, including $C^{2}$ NURBS, they are more commonly applied to individual Bézier curve segments or surface patches).

To date, not many papers have been published that address the rational case. Several papers have been written that study efficient ways to obtain bounds on first derivatives for rational curves and surfaces [Floater 1992; Hermann 1992; Wang et al. 1997] but these techniques are still somewhat costly. Experience with the first derivative suggests that global bounds on second derivatives are either very expensive to compute, or very conservative. Cheng proposed a new method that does not require bounds on the second derivatives [Cheng 1992]. He instead treats a rational surface as a polynomial surface in projective $4 D$ space and shows that, given a tolerance $\epsilon$ and a rational surface $\mathbf{r}(u, v)=\mathbf{R}(u, v) / w(u, v)$, we only need to compute a bound for its associated homogeneous surface $\mathbf{S}(u, v)=(\mathbf{R}(u, v), w(u, v))$ with a new tolerance $\epsilon_{H}$, which guarantees the $\epsilon$-flatness of $\mathbf{r}(u, v)$. The new tolerance is

$$
\epsilon_{H}=\left\{\begin{array}{cl}
\frac{\inf |w(u, v)| \epsilon}{\left(1+(\sup \|\mathbf{r}(u, v)\|-\epsilon)^{2}\right)^{1 / 2}}, & 0 \leq \epsilon \leq \sup \|\mathbf{r}(u, v)\| \\
\inf |w(u, v)| \epsilon, & \sup \|\mathbf{r}(u, v)\|<\epsilon \leq 2 \sup \|\mathbf{r}(u, v)\| \\
+\infty, & 2 \sup \|\mathbf{r}(u, v)\|<\epsilon
\end{array}\right.
$$

Thus the problem of evaluating the bounds on $\mathbf{r}(u, v)$ 's second order derivatives turns to estimating $\mathbf{S}(u, v)$ 's, which is much easier. However, numerical experiments show that this estimation is often too loose.

In this paper, we adhere to Cheng's idea that using homogeneous coordinates for rational curves and surfaces makes derivatives simple, and hence we attempt to bound the maximum deviation based on the associated homogeneous coordinates. Section 2 derives a simple bound expressed by the homogeneous coordinates for 
the distance of two projection points. Then the new step size formulas are developed for rational curves and surfaces in Sections 3 and 4 respectively. It is found that the new bound is tighter than Cheng's. When all the weights of the rational curves or surfaces are the same, the bound reduces to that of polynomial Bézier curves or surfaces. It is expected that the improved bound will lead to more efficient algorithms in graphics and modeling applications.

\section{PROJECTION DISTANCE ESTIMATION}

Our basic strategy is to perform evaluation in homogeneous space. This requires us to approximate the effect of the perspective projection efficiently. The following theorems provide relationships between homogeneous and Euclidean spaces for this purpose. Throughout the paper, $E^{n}$ stands for $n$-dimensional Euclidean space.

THEOREM 1. Let $\mathbf{Q}_{1}=\left(\mathbf{R}_{1}, w_{1}\right)$ and $\mathbf{Q}_{2}=\left(\mathbf{R}_{2}, w_{2}\right)$ with $w_{1}, w_{2}>0$ be two points in $E^{n+1}$, whose corresponding projections are $\mathbf{r}_{1}=\mathbf{R}_{1} / w_{1}, \mathbf{r}_{2}=\mathbf{R}_{2} / w_{2} \in E^{n}$, and let $r$ be any number not smaller than $\max \left(\left\|\mathbf{r}_{1}\right\|,\left\|\mathbf{r}_{2}\right\|\right)$. Then

$$
\left\|\mathbf{r}_{1}-\mathbf{r}_{2}\right\| \leq \frac{\left\|\mathbf{R}_{1}-\mathbf{R}_{2}\right\|+\left(r-\left\|\mathbf{r}_{1}-\mathbf{r}_{2}\right\|\right)\left|w_{1}-w_{2}\right|}{\min \left(w_{1}, w_{2}\right)}
$$

Proof: Without loss of generality, we assume that $w_{1} \leq w_{2}$. Then

$$
\begin{aligned}
\left\|\mathbf{r}_{1}-\mathbf{r}_{2}\right\| & =\left\|\frac{\mathbf{R}_{1}}{w_{1}}-\frac{\mathbf{R}_{2}}{w_{2}}\right\| \leq\left\|\frac{\mathbf{R}_{1}}{w_{1}}-\frac{\mathbf{R}_{1}}{w_{2}}\right\|+\left\|\frac{\mathbf{R}_{1}}{w_{2}}-\frac{\mathbf{R}_{2}}{w_{2}}\right\| \\
& \leq\left\|\frac{\mathbf{R}_{1}}{w_{1}}\right\| \frac{\left|w_{1}-w_{2}\right|}{w_{2}}+\frac{\left\|\mathbf{R}_{1}-\mathbf{R}_{2}\right\|}{w_{2}} \leq r \frac{\left|w_{1}-w_{2}\right|}{w_{2}}+\frac{\left\|\mathbf{R}_{1}-\mathbf{R}_{2}\right\|}{w_{2}} \\
& =\frac{r\left|w_{1}-w_{2}\right|+\left\|\mathbf{R}_{1}-\mathbf{R}_{2}\right\|}{w_{1}+w_{2}-w_{1}}=\frac{r\left|w_{1}-w_{2}\right|+\left\|\mathbf{R}_{1}-\mathbf{R}_{2}\right\|}{\min \left(w_{1}, w_{2}\right)+\left|w_{1}-w_{2}\right|}
\end{aligned}
$$

Thus we obtain

$$
\left(\min \left(w_{1}, w_{2}\right)+\left|w_{1}-w_{2}\right|\right)\left\|\mathbf{r}_{1}-\mathbf{r}_{2}\right\| \leq r\left|w_{1}-w_{2}\right|+\left\|\mathbf{R}_{1}-\mathbf{R}_{2}\right\|
$$

i.e.

Therefore

$$
\min \left(w_{1}, w_{2}\right)\left\|\mathbf{r}_{1}-\mathbf{r}_{2}\right\| \leq\left\|\mathbf{R}_{1}-\mathbf{R}_{2}\right\|+\left(r-\left\|\mathbf{r}_{1}-\mathbf{r}_{2}\right\|\right)\left|w_{1}-w_{2}\right|
$$

$$
\left\|\mathbf{r}_{1}-\mathbf{r}_{2}\right\| \leq \frac{\left\|\mathbf{R}_{1}-\mathbf{R}_{2}\right\|+\left(r-\left\|\mathbf{r}_{1}-\mathbf{r}_{2}\right\|\right)\left|w_{1}-w_{2}\right|}{\min \left(w_{1}, w_{2}\right)}
$$

Theorem 1 has an intuitive geometric interpretation. See Figure 1 for an example in the case of $n=1$. The multiplication of $w_{1}$ and the Euclidean distance of $\mathbf{r}_{1}$ and $\mathbf{r}_{2}$ equals the length of $\mathbf{Q}_{1} \mathbf{I}$, which is the sum of $\left\|\mathbf{Q}_{1} \mathbf{H}\right\|$ and $\|\mathbf{H I}\|$. Note that $\|\mathbf{H I}\|=\left\|\mathbf{Q}_{2} \mathbf{H}\right\| / \tan \alpha,\left\|\mathbf{Q}_{1} \mathbf{H}\right\|$ is $\left\|\mathbf{R}_{1}-\mathbf{R}_{2}\right\|$ and $\left\|\mathbf{Q}_{2} \mathbf{H}\right\|$ is just $\left|w_{1}-w_{2}\right|$. Therefore the inequality (5) is equivalent to $\tan \alpha=\|\mathbf{O C}\| /\left\|\mathbf{r}_{2} \mathbf{C}\right\| \geq 1 /\left(r-\left\|\mathbf{r}_{1}-\mathbf{r}_{2}\right\|\right)$. A similar but looser estimation was given by Tiller [Tiller 1992] and Schaback [Schaback 1993].

TheOREM 2. Let $\mathbf{Q}_{1}=\left(\mathbf{R}_{1}, w_{1}\right)$ and $\mathbf{Q}_{2}=\left(\mathbf{R}_{2}, w_{2}\right)$ be two points in $E^{n+1}$, and let $\epsilon$ and $r$ be two positive numbers. If $\mathbf{Q}_{1}$ and $\mathbf{Q}_{2}$ satisfy

$$
w_{1}, w_{2} \geq 0, \quad \max \left(\left\|\frac{\mathbf{R}_{1}}{w_{1}}\right\|,\left\|\frac{\mathbf{R}_{2}}{w_{2}}\right\|\right) \leq r
$$

and

$$
\frac{\left\|\mathbf{R}_{1}-\mathbf{R}_{2}\right\|+(r-\epsilon)\left|w_{1}-w_{2}\right|}{\min \left(w_{1}, w_{2}\right)} \leq \epsilon
$$

then the distance of their projections $\mathbf{r}_{1}=\mathbf{R}_{1} / w_{1}$ and $\mathbf{r}_{2}=\mathbf{R}_{2} / w_{2}$ is less than or equal to $\epsilon$ :

$$
\left\|\mathbf{r}_{1}-\mathbf{r}_{2}\right\| \leq \epsilon
$$




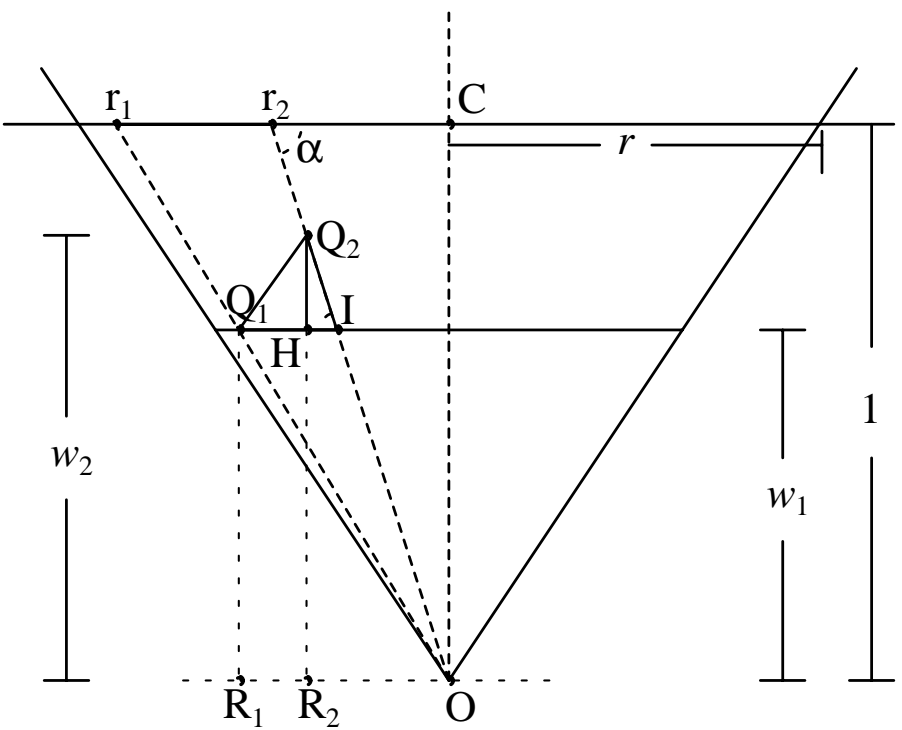

Fig. 1. Geometric interpretation for the case of $n=1$.

Proof: Let $\eta=\left\|\mathbf{r}_{1}-\mathbf{r}_{2}\right\|$. Then

$$
\begin{aligned}
\eta & \leq \frac{\left\|\mathbf{R}_{1}-\mathbf{R}_{2}\right\|+(r-\eta)\left|w_{1}-w_{2}\right|}{\min \left(w_{1}, w_{2}\right)}=\frac{\left\|\mathbf{R}_{1}-\mathbf{R}_{2}\right\|+(r-\epsilon)\left|w_{1}-w_{2}\right|}{\min \left(w_{1}, w_{2}\right)}+\frac{(\epsilon-\eta)\left|w_{1}-w_{2}\right|}{\min \left(w_{1}, w_{2}\right)} \\
& \leq \epsilon+(\epsilon-\eta) \frac{\left|w_{1}-w_{2}\right|}{\min \left(w_{1}, w_{2}\right)}
\end{aligned}
$$

Thus $(\epsilon-\eta)\left(1+\frac{\left|w_{1}-w_{2}\right|}{\min \left(w_{1}, w_{2}\right)}\right) \geq 0$, which arrives at the conclusion: $\epsilon \geq \eta=\left\|\mathbf{r}_{1}-\mathbf{r}_{2}\right\|$.

This theorem shows that the closeness requirement of two points can be imposed on a simple expression represented by the homogeneous coordinates.

\section{STEP SIZE FOR RATIONAL CURVES}

\subsection{General theory}

Since curves have simpler equations, we discuss the curve case first. For a rational curve $\mathbf{r}(t)=\frac{\mathbf{R}(t)}{w(t)}, t \in[0,1]$, the tessellation problem can be formulated: given a tolerance $\epsilon$, find a maximum parameter step $\delta$ such that

$$
\sup _{t \in[\alpha, \beta]}\|\mathbf{r}(t)-\mathbf{L}(t)\| \leq \epsilon
$$

where $\alpha, \beta \in[0,1]$ and $\delta=\beta-\alpha, \mathbf{L}(t)$ is the line segment connecting $\mathbf{r}(\alpha)$ and $\mathbf{r}(\beta)$ parameterized as follows:

$$
\mathbf{L}(t)=\frac{\mathbf{L}_{n}(t)}{L_{d}(t)}=\frac{[(\beta-t) \mathbf{R}(\alpha)+(t-\alpha) \mathbf{R}(\beta)] /(\beta-\alpha)}{[(\beta-t) w(\alpha)+(t-\alpha) w(\beta)] /(\beta-\alpha)}
$$

In practice, we typically content ourselves with finding a step size for which (9) is an upper bound. Surprisingly, situations exist for which a step size $\delta_{1}<\delta$ can actually violate the $\epsilon$-error, even though a step size of $\delta$ does not. Figure 2 illustrates such a case.

In light of this problem, we modify our definition of $\delta$ as follows:

$$
\delta=\sup \left\{\rho \mid \sup _{t \in[\alpha, \alpha+\eta]}\|\mathbf{r}(t)-\mathbf{L}(t)\| \leq \epsilon \text { for any positive } \eta \leq \rho, \quad \alpha, \alpha+\rho \in[0,1]\right\}
$$

With Theorem 2, we need to compute a bound for $\left\|\mathbf{R}(t)-\mathbf{L}_{n}(t)\right\|+(r-\epsilon)\left|w(t)-L_{d}(t)\right|$. The tighter the bound, the larger (and the more economical) the step size $\delta$. The formula (1) can be simply used here by finding the bounds on $\left\|\mathbf{R}^{\prime \prime}(t)\right\|$ and $\left|w^{\prime \prime}(t)\right|$, and combining them. However, this is different from taking 


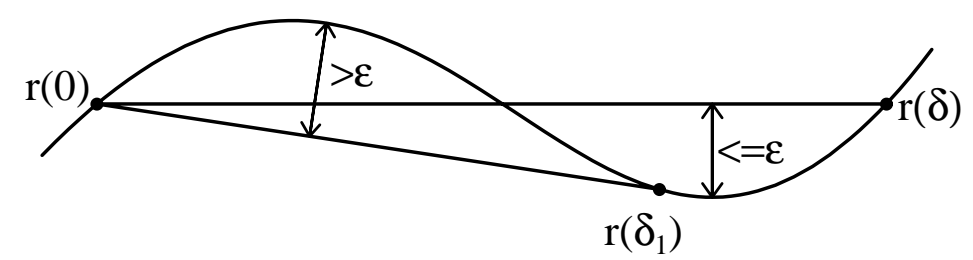

Fig. 2. Line $\mathbf{r}(0) \mathbf{r}\left(\delta_{1}\right)$ is not within $\epsilon$-flatness while $\mathbf{r}(0) \mathbf{r}(\delta)$ is.

$\left\|\mathbf{R}^{\prime \prime}(t)\right\|+(r-\epsilon)\left|w^{\prime \prime}(t)\right|$ as a whole. In general, the latter is smaller than the former since the maxima for $\left\|\mathbf{R}^{\prime \prime}(t)\right\|$ and $\left|w^{\prime \prime}(t)\right|$ may occur at different parameter values. In the following we show that the proof of Theorem 2 in [Filip et al. 1986] can be generalized for this.

Lemma 1. Let $\mathbf{Q}(t)=(\mathbf{R}(t), w(t)) \in E^{n+1}$ be a $C^{2}$ vector-valued function defined over $[\alpha, \beta]$ with $\mathbf{Q}(\alpha)=$ $\mathbf{Q}(\beta)=0$. Let $k$ be any nonnegative number. Then

$$
\sup _{t \in[\alpha, \beta]}(\|\mathbf{R}(t)\|+k|w(t)|) \leq \frac{(\beta-\alpha)^{2}}{8} \sup _{t \in[\alpha, \beta]}\left(\left\|\mathbf{R}^{\prime \prime}(t)\right\|+k\left|w^{\prime \prime}(t)\right|\right)
$$

Proof: Let $d(t)=\|\mathbf{R}(t)\|+k|w(t)|$. Then $d(t)$ is a continuous function in $[\alpha, \beta]$ with $d(\alpha)=d(\beta)=0$ and $d(t) \geq 0$, which means $d(t)$ can attain its maximum value at some $\xi \in(\alpha, \beta)$. It is obvious that $d(t)$ is $C^{2}$ continuous in $[\alpha, \beta]$ possibly except those points where $w(t)=0$ or $\mathbf{R}(t)=0$. Now we discuss the problem in three cases:

case $1 . w(\xi)=0$.

In this case, $d(\xi)=\|\mathbf{R}(\xi)\|$. From (1),

$$
\|\mathbf{R}(\xi)\| \leq \frac{(\beta-\alpha)^{2}}{8} \sup _{t \in[\alpha, \beta]}\left\|\mathbf{R}^{\prime \prime}(t)\right\|
$$

Let $\eta$ be so that $\left\|\mathbf{R}^{\prime \prime}(\eta)\right\|=\sup _{t \in[\alpha, \beta]}\left\|\mathbf{R}^{\prime \prime}(t)\right\|$. We have

$$
\begin{aligned}
d(\xi) & =\|\mathbf{R}(\xi)\| \leq \frac{(\beta-\alpha)^{2}}{8}\left\|\mathbf{R}^{\prime \prime}(\eta)\right\| \\
& \leq \frac{(\beta-\alpha)^{2}}{8}\left(\left\|\mathbf{R}^{\prime \prime}(\eta)\right\|+k\left|w^{\prime \prime}(\eta)\right|\right) \leq \frac{(\beta-\alpha)^{2}}{8} \sup _{t \in[\alpha, \beta]}\left(\left\|\mathbf{R}^{\prime \prime}(\eta)\right\|+k\left|w^{\prime \prime}(\eta)\right|\right)
\end{aligned}
$$

case 2 . $\mathbf{R}(\xi)=0$.

Then $d(\xi)=k|w(\xi)|$. By a like argument as in case 1 , we obtain

$$
d(\xi)=\frac{(\beta-\alpha)^{2}}{8} k \sup _{t \in[\alpha, \beta]}\left|w^{\prime \prime}(t)\right| \leq \frac{(\beta-\alpha)^{2}}{8} \sup _{t \in[\alpha, \beta]}\left(\left\|\mathbf{R}^{\prime \prime}(t)\right\|+k\left|w^{\prime \prime}(t)\right|\right)
$$

case $3 . w(\xi) \neq 0, \mathbf{R}(\xi) \neq 0$.

Thus $d(t)$ is $C^{2}$ at $t=\xi$ and $d^{\prime}(\xi)=0$, i.e.

$$
d^{\prime}(\xi)=\frac{\mathbf{R}}{\|\mathbf{R}\|} \mathbf{R}^{\prime}(\xi)+k \frac{w}{|w|} w^{\prime}(\xi)=0
$$

Now we express $\mathbf{Q}(t)$ in its Taylor expansion about $\xi$ :

$$
(\mathbf{R}(t), w(t))=(\mathbf{R}(\xi), w(\xi))+\left(\mathbf{R}^{\prime}(\xi), w^{\prime}(\xi)\right)(t-\xi)+\int_{\xi}^{t}\left(\mathbf{R}^{\prime \prime}(s), w^{\prime \prime}(s)\right)(t-s) d s
$$


Without loss of generality, we assume that $\xi \in\left[\frac{\alpha+\beta}{2}, \beta\right)$. Then substituting $t=\beta$ into the equation (13) yields

$$
0=(\mathbf{R}(\beta), w(\beta))=(\mathbf{R}(\xi), w(\xi))+\left(\mathbf{R}^{\prime}(\xi), w^{\prime}(\xi)\right)(\beta-\xi)+\int_{\xi}^{\beta}\left(\mathbf{R}^{\prime \prime}(s), w^{\prime \prime}(s)\right)(\beta-s) d s
$$

Taking the dot product of equation (14) with $\left(\frac{\mathbf{R}(\xi)}{\|\mathbf{R}(\xi)\|}, k \frac{w(\xi)}{|w(\xi)|}\right)$, we obtain

$$
0=\|\mathbf{R}(\xi)\|+k|w(\xi)|+0+\int_{\xi}^{\beta}\left(\frac{\mathbf{R}(\xi) \mathbf{R}^{\prime \prime}(s)}{\|\mathbf{R}(\xi)\|}+k \frac{w(\xi) w^{\prime \prime}(s)}{|w(\xi)|}\right)(\beta-s) d s
$$

Thus

$$
\begin{aligned}
d(\xi) & =\|\mathbf{R}(\xi)\|+k|w(\xi)|=\left|\int_{\xi}^{\beta}\left(\frac{\mathbf{R}(\xi) \mathbf{R}^{\prime \prime}(s)}{\|\mathbf{R}(\xi)\|}+k \frac{w(\xi) w^{\prime \prime}(s)}{|w(\xi)|}\right)(\beta-s) d s\right| \\
& \leq \int_{\xi}^{\beta}\left(\left|\frac{\mathbf{R}(\xi) \mathbf{R}^{\prime \prime}(s)}{\|\mathbf{R}(\xi)\|}\right|+k\left|\frac{w(\xi) w^{\prime \prime}(s)}{|w(\xi)|}\right|\right)(\beta-s) d s \leq \int_{\xi}^{\beta}\left(\left\|\mathbf{R}^{\prime \prime}(s)\right\|+k\left|w^{\prime \prime}(s)\right|\right)(\beta-s) d s \\
& \leq \sup _{t \in[\alpha, \beta]}\left(\left\|\mathbf{R}^{\prime \prime}(t)\right\|+k\left|w^{\prime \prime}(t)\right|\right) \int_{\xi}^{\beta}(\beta-s) d s=\sup _{t \in[\alpha, \beta]}\left(\left\|\mathbf{R}^{\prime \prime}(t)\right\|+k\left|w^{\prime \prime}(t)\right|\right) \frac{(\beta-\xi)^{2}}{2} \\
& \leq \frac{(\beta-\alpha)^{2}}{8} \sup _{t \in[\alpha, \beta]}\left(\left\|\mathbf{R}^{\prime \prime}(t)\right\|+k\left|w^{\prime \prime}(t)\right|\right)
\end{aligned}
$$

Now we are ready to derive the formula for the step size.

ThEOREM 3. Given a $C^{2}$ rational curve $\mathbf{r}(t)=\frac{\mathbf{R}(t)}{w(t)}, t \in[\alpha, \beta]$ with $w(t)>0$, and $r$ is a number satisfying $r \geq \sup _{t \in[\alpha, \beta]}\|\mathbf{r}(t)\|=\sup _{t \in[\alpha, \beta]}\left\|\frac{\mathbf{R}(t)}{w(t)}\right\|$. Let $\mathbf{L}(t)=\frac{\mathbf{L}_{n}(t)}{L_{d}(t)}$ be defined as in (10), then $\sup _{t \in[\alpha, \beta]}\|\mathbf{r}(t)-\mathbf{L}(t)\| \leq \epsilon$ if

$$
\delta=\beta-\alpha \leq\left\{\begin{array}{cc}
\sqrt{\frac{8 \inf _{t}\{w(t)\} \epsilon}{\sup _{t}\left(\left\|\mathbf{R}^{\prime \prime}(t)\right\|+(r-\epsilon)\left|w^{\prime \prime}(t)\right|\right)},} & \epsilon<r \\
\sqrt{\frac{8 \inf _{t}\{w(t)\} \epsilon}{\sup _{t}\left\|\mathbf{R}^{\prime \prime}(t)\right\|},} & r \leq \epsilon<2 r \\
1, & 2 r \leq \epsilon
\end{array}\right.
$$

Proof: First let us consider the case of $2 r \leq \epsilon$. Since

$$
\mathbf{L}(t)=\frac{\mathbf{R}(\alpha)(\beta-t)+\mathbf{R}(\beta)(t-\alpha)}{w(\alpha)(\beta-t)+w(\beta)(t-\alpha)}=\frac{\mathbf{R}(\alpha)}{w(\alpha)} \frac{(\beta-t) w(\alpha)}{(\beta-t) w(\alpha)+(t-\alpha) w(\beta)}+\frac{\mathbf{R}(\beta)}{w(\beta)} \frac{(t-\alpha) w(\beta)}{(\beta-t) w(\alpha)+(t-\alpha) w(\beta)},
$$

$\|\mathbf{L}(t)\| \leq \max \left(\left\|\frac{\mathbf{R}(\alpha)}{w(\alpha)}\right\|,\left\|\frac{\mathbf{R}(\beta)}{w(\beta)}\right\|\right) \leq r$. Then for any $t \in[\alpha, \beta],\|\mathbf{r}(t)-\mathbf{L}(t)\| \leq\|\mathbf{r}(t)\|+\|\mathbf{L}(t)\| \leq r+r=2 r \leq \epsilon$.

Second, in the case of $r \leq \epsilon<2 r$, applying (1) to $\mathbf{R}(t)$, we get

$$
\sup _{t \in[\alpha, \beta]}\left\|\mathbf{R}(t)-\mathbf{L}_{n}(t)\right\| \leq \frac{(\beta-\alpha)^{2}}{8} \sup _{t \in[\alpha, \beta]}\left\|\mathbf{R}^{\prime \prime}(t)\right\|
$$

As $L_{d}(t)$ is a convex combination of $w(\alpha)$ and $w(\beta)$, the value of $L_{d}(t)$ is between $w(\alpha)$ and $w(\beta)$. Hence

$$
\sup _{t \in[\alpha, \beta]} \frac{\left\|\mathbf{R}(t)-\mathbf{L}_{n}(t)\right\|+(r-\epsilon)\left|w(t)-L_{d}(t)\right|}{\min \left(w(t), L_{d}(t)\right)} \leq \sup _{t \in[\alpha, \beta]} \frac{\left\|\mathbf{R}(t)-\mathbf{L}_{n}(t)\right\|}{\min \left(w(t), L_{d}(t)\right)} \leq \frac{\delta^{2}}{8} \frac{\sup _{t \in[\alpha, \beta]}\left\|\mathbf{R}^{\prime \prime}(t)\right\|}{\inf _{t \in[\alpha, \beta]} w(t)} \leq \epsilon
$$

By Theorem 2, $\sup _{t \in[\alpha, \beta]}\|\mathbf{r}(t)-\mathbf{L}(t)\| \leq \epsilon$.

Finally, for the case of $\epsilon<r, r-\epsilon>0$. Note that $\mathbf{R}(\alpha)-\mathbf{L}_{n}(\alpha)=\mathbf{R}(\beta)-\mathbf{L}_{n}(\beta)=0$ and $w(\alpha)-L_{d}(\alpha)=$ $w(\beta)-L_{d}(\beta)=0$. From Lemma 1 ,

$$
\sup _{t \in[\alpha, \beta]}\left(\left\|\mathbf{R}(t)-\mathbf{L}_{n}(t)\right\|+(r-\epsilon)\left|w(t)-L_{d}(t)\right|\right) \leq \frac{(\beta-\alpha)^{2}}{8} \sup _{t \in[\alpha, \beta]}\left(\left\|\mathbf{R}^{\prime \prime}(t)\right\|+(r-\epsilon)\left|w^{\prime \prime}(t)\right|\right)
$$


So

$$
\sup _{t \in[\alpha, \beta]} \frac{\left\|\mathbf{R}(t)-\mathbf{L}_{n}(t)\right\|+(r-\epsilon)\left|w(t)-L_{d}(t)\right|}{\min \left(w(t), L_{d}(t)\right)} \leq \frac{\sup _{t \in[\alpha, \beta]}\left(\left\|\mathbf{R}^{\prime \prime}(t)\right\|+(r-\epsilon)\left|w^{\prime \prime}(t)\right|\right)}{\inf _{t} w(t)} \frac{(\beta-\alpha)^{2}}{8} \leq \epsilon
$$

Thus the proof is completed by Theorem 2 .

\subsection{Rational Bezier curves}

For a rational Bézier curve $\mathbf{r}(t)$ defined by

$$
\mathbf{r}(t)=\frac{\mathbf{R}(t)}{w(t)}=\frac{\sum_{i=0}^{n} \mathbf{P}_{i} w_{i} B_{i}^{n}(t)}{\sum_{i=0}^{n} w_{i} B_{i}^{n}(t)}, t \in[0,1]
$$

$\mathbf{R}(t)$ and $w(t)$ are in Bézier form. The estimation for the sup's of their second order derivatives and for inf $\{w(t)\}$ is straightforward due to the convex-hull property of polynomials in Bernstein form. Define $\Delta$ to be a forward difference operator so that $\Delta P_{i}=P_{i+1}-P_{i}$. We have

Corollary 1. Let $\mathbf{r}(t)$ be a rational Bézier curve (16), $r=\max _{i}\left\|\mathbf{P}_{i}\right\|$ and $w=\min _{i}\left\{w_{i}\right\}>0$. For any $\epsilon>0$, the deviation of the curve segment over $[\alpha, \beta] \subseteq[0,1]$ from its fractional linear approximation is within $\epsilon$, i.e. $\sup _{t \in[\alpha, \beta]}\|\mathbf{r}(t)-\mathbf{L}(t)\| \leq \epsilon$ if $\delta=\beta-\alpha$ satisfies $t \in[\alpha, \beta]$

$$
\delta \leq\left\{\begin{array}{cc}
\sqrt{\frac{8 w \epsilon}{n(n-1) \max _{i}\left(\left\|\Delta^{2}\left(w_{i} \mathbf{P}_{i}\right)\right\|+(r-\epsilon)\left|\Delta^{2} w_{i}\right|\right)}}, & \epsilon<r \\
\sqrt{\frac{8 w \epsilon}{n(n-1) \max _{i}\left\|\Delta^{2}\left(w_{i} \mathbf{P}_{i}\right)\right\|}}, & r \leq \epsilon<2 r \\
1, & 2 r \leq \epsilon
\end{array}\right.
$$

Proof: The second order derivative of a Bézier curve is also a Bézier curve with degree lower by 2 , e.g.

$$
\left(\mathbf{R}^{\prime \prime}(t), w^{\prime \prime}(t)\right)=n(n-1) \sum_{i=0}^{n-2} \Delta^{2}\left(w_{i} \mathbf{P}_{i}, w_{i}\right) B_{i}^{n-2}(t)
$$

The conclusion follows immediately from the inequalities

$$
\left\|\mathbf{R}^{\prime \prime}(t)\right\| \leq n(n-1) \sum_{i=0}^{n-2}\left\|\Delta^{2}\left(w_{i} \mathbf{P}_{i}\right)\right\| B_{i}^{n-2}(t) \leq n(n-1) \max _{i}\left\|\Delta^{2}\left(w_{i} \mathbf{P}_{i}\right)\right\|
$$

and

$$
\begin{aligned}
\left\|\mathbf{R}^{\prime \prime}(t)\right\|+(r-\epsilon)\left|w^{\prime \prime}(t)\right| & \leq n(n-1) \sum_{i=0}^{n-2}\left(\left\|\Delta^{2}\left(w_{i} \mathbf{P}_{i}\right)\right\|+(r-\epsilon)\left|\Delta^{2} w_{i}\right|\right) B_{i}^{n-2}(t) \\
& \leq n(n-1) \max _{i}\left(\left\|\Delta^{2}\left(w_{i} \mathbf{P}_{i}\right)\right\|+(r-\epsilon)\left|\Delta^{2} w_{i}\right|\right)
\end{aligned}
$$

It is possible for one of the $w_{i}$ to be zero, but still have a well-defined curve. In this case, a better estimation for $\inf \{w(t)\}$ can be obtained by first degree elevating or subdividing the polynomial $w(t)$ and then taking $\min \left\{w_{i}\right\}$ of the resulting weights.

\section{Examples.}

(1) Quadratic rational Bézier curve: the step size for the case of $\epsilon<r$ is

$$
\delta=\sqrt{\frac{4 \min \left\{w_{0}, w_{1}, w_{2}\right\} \epsilon}{\left\|w_{2} \mathbf{P}_{2}-2 w_{1} \mathbf{P}_{1}+w_{0} \mathbf{P}_{0}\right\|+(r-\epsilon)\left|w_{2}-2 w_{1}+w_{0}\right|}}
$$


(2) Cubic rational Bézier curve: the step size for the case of $\epsilon<r$ is

$$
\delta=\sqrt{\frac{4 \min \left\{w_{0}, w_{1}, w_{2}, w_{3}\right\} \epsilon}{3 \max \left\{\left\|\Delta^{2}\left(w_{0} \mathbf{P}_{0}\right)\right\|+(r-\epsilon)\left|\Delta^{2} w_{0}\right|,\left\|\Delta^{2}\left(w_{1} \mathbf{P}_{1}\right)\right\|+(r-\epsilon)\left|\Delta^{2} w_{1}\right|\right\}}}
$$

\section{Remarks.}

(1) Here we show that the new step size $\delta_{n}$ given by (17) is larger than Cheng's. For a rational Bézier curve, Cheng's step size is

$$
\delta_{c}=\sqrt{\frac{8 \epsilon_{H}}{n(n-1) \max _{i}\left\|\Delta^{2}\left(w_{i} \mathbf{P}_{i}, w_{i}\right)\right\|}}
$$

and $\epsilon_{H}$ is defined in (4). When $\epsilon<r$,

$$
\begin{aligned}
\left(8 w \epsilon / n(n-1) \delta_{c}^{2}\right)^{2} & =\left(1+(r-\epsilon)^{2}\right) \max _{i}\left(\left\|\Delta^{2}\left(w_{i} \mathbf{P}_{i}\right)\right\|^{2}+\left|\Delta^{2} w_{i}\right|^{2}\right) \\
& =\max _{i}\left(\left\|\Delta^{2}\left(w_{i} \mathbf{P}_{i}\right)\right\|^{2}+(r-\epsilon)^{2}\left|\Delta^{2} w_{i}\right|^{2}+(r-\epsilon)^{2}\left\|\Delta^{2}\left(w_{i} \mathbf{P}_{i}\right)\right\|^{2}+\left|\Delta^{2} w_{i}\right|^{2}\right) \\
& \geq \max _{i}\left(\left\|\Delta^{2}\left(w_{i} \mathbf{P}_{i}\right)\right\|^{2}+(r-\epsilon)^{2}\left|\Delta^{2} w_{i}\right|^{2}+2(r-\epsilon)\left\|\Delta^{2}\left(w_{i} \mathbf{P}_{i}\right)\right\|\left|\Delta^{2} w_{i}\right|\right) \\
& =\max _{i}\left(\left\|\Delta^{2}\left(w_{i} \mathbf{P}_{i}\right)\right\|+(r-\epsilon)\left|\Delta^{2} w_{i}\right|\right)^{2}=\left(8 w \epsilon / n(n-1) \delta_{n}^{2}\right)^{2}
\end{aligned}
$$

so $\delta_{n} \geq \delta_{c}$. The other cases are trivial.

(2) When all weights are identical, the rational Bézier curve reduces to a polynomial curve

$$
\mathbf{r}(t)=\sum_{i=0}^{n} \mathbf{P}_{i} B_{i}^{n}(t), \quad t \in[0,1]
$$

In this case $\Delta^{2} w_{i}=0$, so the new step size is the same as that for a Bézier curve, which was given in [Filip et al. 1986], i.e.

$$
\delta \leq \sqrt{\frac{8 \epsilon}{n(n-1) \max _{i}\left\|\Delta^{2} \mathbf{P}_{i}\right\|}}
$$

However Cheng's estimation, in general, does not reduce to — and is more conservative than - (21).

\subsection{Further improvements}

Further improvements without much additional computation are possible. The first observation is that the bound for the second derivative curve (18) might be tightened. Farin points out that a rational curve can be bounded in a tighter convex hull generated by the end points and so-called "weight points" [Farin 1993]. The weight points are obtained after an additional subdivision without doing the full work of subdividing. In our case, the weight points are just the average of the adjacent homogeneous points. That is

$$
\mathbf{W}_{i}=\left(\mathbf{W R}_{i}, W w_{i}\right)=n(n-1) \frac{\Delta^{2}\left(w_{i-1} \mathbf{P}_{i-1}, w_{i-1}\right)+\Delta^{2}\left(w_{i} \mathbf{P}_{i}, w_{i}\right)}{2}, i=1 \cdots n-2
$$

If we also denote the end points by $\mathbf{W}_{0}=n(n-1) \Delta^{2}\left(w_{0} \mathbf{P}_{0}, w_{0}\right)$ and $\mathbf{W}_{n-1}=n(n-1) \Delta^{2}\left(w_{n-2} \mathbf{P}_{n-2}, w_{n-2}\right)$, then the curve (18) lies in the convex hull that includes only $\mathbf{W}_{i}(i=0, \cdots, n-1)$. Therefore for any $t \in[0,1]$, there exist nonnegative coefficients $\lambda_{i}(t)$ such that

$$
\left(\mathbf{R}^{\prime \prime}(t), w^{\prime \prime}(t)\right)=\sum_{i=0}^{n-1} \lambda_{i}(t) \mathbf{W}_{i}, \quad \lambda_{0}(t)+\lambda_{1}(t)+\cdots+\lambda_{n-1}(t)=1
$$


Therefore

$$
\begin{aligned}
\left\|\mathbf{R}^{\prime \prime}(t)\right\| & \leq \sum_{i=0}^{n-1}\left\|\mathbf{W} \mathbf{R}_{i}\right\| \lambda_{i}(t) \leq \max _{i}\left\|\mathbf{W} \mathbf{R}_{i}\right\| \\
& =n(n-1) \max _{i}\left\{\left\|\Delta^{2}\left(w_{0} \mathbf{P}_{0}\right)\right\|, \frac{\left\|\Delta^{2}\left(w_{i-1} \mathbf{P}_{i-1}\right)+\Delta^{2}\left(w_{i} \mathbf{P}_{i}\right)\right\|}{2},\left\|\Delta^{2}\left(w_{n-2} \mathbf{P}_{n-2}\right)\right\|\right\}
\end{aligned}
$$

and

$$
\begin{aligned}
\left\|\mathbf{R}^{\prime \prime}(t)\right\|+(r-\epsilon)\left|w^{\prime \prime}(t)\right| \leq & \sum_{i=0}^{n-1}\left(\left\|\mathbf{W} \mathbf{R}_{i}\right\|+(r-\epsilon)\left|W w_{i}\right|\right) \lambda_{i}(t) \\
\leq & \max _{i}\left(\left\|\mathbf{W} \mathbf{R}_{i}\right\|+(r-\epsilon)\left|W w_{i}\right|\right) \\
& =n(n-1) \max _{i}\left\{\left\|\Delta^{2}\left(w_{0} \mathbf{P}_{0}\right)\right\|+(r-\epsilon)\left|\Delta^{2} w_{0}\right|,\right. \\
& \frac{\left\|\Delta^{2}\left(w_{i-1} \mathbf{P}_{i-1}\right)+\Delta^{2}\left(w_{i} \mathbf{P}_{i}\right)\right\|}{2}+(r-\epsilon) \frac{\left|\Delta^{2} w_{i-1}+\Delta^{2} w_{i}\right|}{2}, \\
& \left.\left\|\Delta^{2}\left(w_{n-2} \mathbf{P}_{n-2}\right)\right\|+(r-\epsilon)\left|\Delta^{2} w_{n-2}\right|\right\}
\end{aligned}
$$

Usually this gives a tighter bound. The improvement depends on the distribution of the control points of curve (18). If the adjacent control points are close, the averages formed will not be much less in magnitude than the original ones. For a cubic rational curve, (18) is a linear polynomial, in which case there is no improvement in using the weight-point method.

The second observation is that translation does not change the shape of the curve and the translated curve should have the same step size as the original curve. However the formula (15) or (17) is dependent on the affine coordinate system. This suggests that we move the original point of the affine coordinate system to make the value of $r=\max _{i}\left\|\mathbf{P}_{i}\right\|$ as small as possible. One simple way is to find the min-max bounding box of the rational curve and to choose the center of the box as the origin of a local coordinate system, or we can find a bounding circle or sphere and then use its center as the origin. The method proposed above can be applied to these control points in their local coordinate system, which also means that the tessellation will be invariant to translation. It should be pointed out that this is just a heuristic approach. When the control points are moved, the values of $\left\|\Delta^{2}\left(w_{i} \mathbf{P}_{i}\right)\right\|$ are also changed. So sometimes the result might be worse. But the numerical examples indicate that in most cases doing a certain translation gives a better step size, especially when the control points are located very asymmetrically around the original point.

Summarizing, we have the following algorithm:

Input: the control points $P_{i}$ and weights $w_{i}$, the error tolerance $\epsilon$.

Output: a global step size

Procedure:

Step 1. Find a min-max bounding box of the curve, and compute its center point, denoted by $C$.

Step 2. Translate the control points by $C: \quad P_{i} \leftarrow P_{i}-C$.

Step 3. Compute the Bézier representation (18) for the second derivative of the homogeneous Bézier curve.

Step 4. Compute $\sup \left(\left\|\mathbf{R}^{\prime \prime}(t)\right\|+(r-\epsilon)\left|w^{\prime \prime}(t)\right|\right)$ or $\sup \left\|\mathbf{R}^{\prime \prime}(t)\right\|$ by formula (24) or (23).

Step 5. compute the step size $\delta$ by $(15)$. 


\subsection{Numerical experiments}

We present a few planar curve examples. The data defining the rational Bézier curves are generated randomly. $C_{i}(t)$ represents the curve of degree $i$.

$$
\begin{aligned}
C_{1}(t): & (2,5,5.6),(1,8,0.7) \\
C_{2}(t): & (-3,-10,0.96),(6,8,2.3),(2,4,0.63) \\
C_{3}(t): & (19,61,0.08),(-61,52,0.5),(17,55,1),(49,-20,0.4) \\
C_{4}(t): & (1,5,6.1),(7,7,0.39),(-8,-10,18.4),(-1,-10,1.1),(-6,-3,0.03) \\
C_{5}(t): & (53,-6,0.7),(-7,66,1.8),(-64,-46,147),(-71,43,6.6),(97,-68,4),(-66,57,0.7) \\
C_{6}(t): & (36,-23,1.7),(48,54,0.8),(14,-13,0.2),(64,13,1),(-68,54,1.4),(43,-1,0.4),(34,92,0.2) \\
C_{7}(t): & (9,9,1.5),(-4,0,3.1),(5,0,3.3),(-7,0,2.7),(10,-10,2.6),(2,-3,0.7), \\
& (-4,9,1.1),(1,-5,1.3) \\
C_{8}(t): & (3,2,0.2),(-5,8,1.6),(1,4,0.8),(7,10,0.8),(1,-8,1.1),(-7,5,1.3),(0,10,0.4), \\
& (4,1,0.7),(6,-1,2.3)
\end{aligned}
$$

In each tuple, the first two numbers stand for the control point's $x, y$-components, and the third for the weight. Different methods are used to compute the step size $\delta$ with the tolerance $\epsilon=0.1$. The results are listed in Table 1 . Here, we test the new methods with just using formula (17), or using weight-point method, or translating the control points first and then using (17), or translating the control points first and then using weight-point method. They are respectively denoted by "new", "new-w", "new-t" and "new-tw". Cheng's method is labelled "cheng". A well-known method for finding a step in a parametric curve is

$$
\delta=\sqrt{\frac{8 \epsilon}{D}}
$$

where $D$ is an upper bound on the magnitude of the second derivative vector of the curve. Variations of this step size are discussed in [Wang et al. 1997; Filip et al. 1986]. For a rational curve of degree $n$, the second derivative vector is degree $3 n$. For comparison with the new method, we computed in two different ways a step size using the method in (25). First, we expressed the second derivative in rational Bernstein form and took $D$ to be the largest distance from the origin to any control point. This method is labelled "approx-D" in the table. We also went to the expense of computing the tight upper bound on the second derivative curve. This amounted to finding the global maximum of a degree $6 n$ polynomial over the $[0,1]$ interval, a computation that is far too costly for practical use, but which is of interest because it yields the largest step size of which (25) is capable. The step size produced in this way is labelled "exact-D" in the table.

\begin{tabular}{|c||l|l|l|l|l|l|l|l|}
\hline$\delta$ & $C_{1}(t)$ & $C_{2}(t)$ & $C_{3}(t)$ & $C_{4}(t)$ & $C_{5}(t)$ & $C_{6}(t)$ & $C_{7}(t)$ & $C_{8}(t)$ \\
\hline \hline cheng & 1.0 & 0.017 & 0.0003 & 0.0001 & 0.00008 & 0.00018 & 0.0027 & 0.0012 \\
\hline new & 1.0 & 0.0549 & 0.0075 & 0.0015 & 0.0007 & 0.0035 & 0.0108 & 0.0072 \\
\hline new-w & 1.0 & 0.0549 & 0.0075 & 0.0019 & 0.00098 & 0.0048 & 0.0132 & 0.0072 \\
\hline new-t & 1.0 & 0.0551 & 0.0081 & 0.0016 & 0.0007 & 0.0037 & 0.0111 & 0.0075 \\
\hline new-tw & 1.0 & 0.0551 & 0.0081 & 0.0021 & 0.001 & 0.0052 & 0.0132 & 0.0075 \\
\hline approx-D & 0.048 & 0.0335 & 0.0039 & 0.0015 & 0.001 & 0.0052 & 0.0121 & 0.0032 \\
\hline exact-D & 0.085 & 0.0457 & 0.0039 & 0.0019 & 0.0013 & 0.0058 & 0.0130 & 0.0036 \\
\hline
\end{tabular}

Table 1. The step sizes computed by different methods with $\epsilon=0.1$.

We also ran several sets of numerical comparisons on several hundred randomly generated test cases of degree three and four. In one batch of tests, the $(x, y)$ coordinates of the control points were generated as random numbers in the interval $([-1000,1000],[-1000,1000])$. We also ran tests in the intervals $([-100,100],[-100,100])$ and $([-10,10],[-10,10])$. Control point weights were generated as the ratio of two random numbers in the intervals $[1,10000]$, and $\epsilon$ was fixed at 0.1 . In each batch, we ran several hundred test cases, computed the ratio between the "new-tw" step size, and those produced by Cheng's method and by the "approx-D" method. The results are displayed in Table 2 .

Two things are noteworthy in this table. First, it is somewhat surprising that "new-tw" gives on average twice as large of a step size as "approx-D". This can be explained. In the case of a polynomial Bézier curve, it is easily shown that the two methods will give exactly the same step size. However, if the weights vary significantly, 


\begin{tabular}{|l|l|l|l|}
\hline Interval & Degree & $\frac{\text { new-tw }}{\text { cheng }}$ & $\frac{\text { new-tw }}{\text { approx }-D}$ \\
\hline \hline$([-1000,1000],[-1000,1000])$ & 3 & 66.4 & 2.03 \\
\hline$([-1000,1000],[-1000,1000])$ & 4 & 72.6 & 1.95 \\
\hline$([-100,100],[-100,100])$ & 3 & 20.2 & 1.92 \\
\hline$([-10,10],[-10,10])$ & 3 & 6.8 & 2.56 \\
\hline
\end{tabular}

Table 2. Average ratio of step sizes with $\epsilon=0.1$.

"new-tw" is often able to provide a larger step size than "approx-D" because it is finding the distance between a rational curve segment and a fractional-linearly parametrized line segment. By contrast, "approx-D" finds the distance between the rational curve segment and a linearly-parametrized line segment.

The second curiosity in Table 2 is that Cheng's method appears to improve as the size of the control point coordinates decreases! Inspection of Cheng's formulae confirms that this should indeed occur. Furthermore, it can be seen that Cheng's method will produce a different step size even if the control points and $\epsilon$ are scaled the same amount! This suggests that Cheng's method might be able to produce a larger step size if we first scale the control points and $\epsilon$ by some constant, and that there might be an optimal scale factor that will produce an optimally large step size. Closer study reveals that, in fact, our step size is exactly the one produced by Cheng's method after applying the optimal scaling!

\section{STEP SIZES FOR RATIONAL SURFACES}

The development for rational surfaces parallels that for rational curves. In this section we just list the results without proofs except the following lemma. Of course, when tessellating two adjacent patches, it is a good idea to compute step sizes for all four boundary curves independently from the step size for the patch interior to avoid cracks (see [Rockwood et al. 1989]).

Lemma 2. Let $\mathbf{Q}(u, v)=(\mathbf{R}(u, v), w(u, v))$ be a $C^{2}$ vector-valued function defined over a right triangle $T$ with vertices $A, B$ and $C$ of the form $B=A+\left(\delta_{u}, 0\right)$ and $C=A+\left(0, \delta_{v}\right)$, and vanish at these three vertices $\mathbf{Q}(A)=\mathbf{Q}(B)=\mathbf{Q}(C)=0$. Then for any nonnegative number $k$,

$$
\sup _{(u, v) \in T}(\|\mathbf{R}(u, v)\|+k|w(u, v)|) \leq\left(D_{u u} \delta_{u}^{2}+2 D_{u v} \delta_{u} \delta_{v}+D_{v v} \delta_{v}^{2}\right) / 8
$$

where

$$
\begin{aligned}
& D_{u u}=\sup _{(u, v) \in T}\left(\left\|\mathbf{R}_{u u}^{\prime \prime}(u, v)\right\|+k\left|w_{u u}^{\prime \prime}(u, v)\right|\right) \\
& D_{u v}=\sup _{(u, v) \in T}\left(\left\|\mathbf{R}_{u v}^{\prime \prime}(u, v)\right\|+k\left|w_{u v}^{\prime \prime}(u, v)\right|\right) \\
& D_{v v}=\sup _{(u, v) \in T}\left(\left\|\mathbf{R}_{v v}^{\prime \prime}(u, v)\right\|+k\left|w_{v v}^{\prime \prime}(u, v)\right|\right)
\end{aligned}
$$

Proof: This lemma can be proven in a way similar to that in [Filip et al. 1986]. Let $D(u, v)=\|\mathbf{R}(u, v)\|+$ $k|w(u, v)| . D(u, v)$ is a continuous function and $D(u, v) \geq 0, D(A)=D(B)=D(C)=0$. Then $D(u, v)$ must get its maximum value at some point $P_{0}=\left(u_{0}, v_{0}\right)$ in $T$. Suppose $P_{0}$ lies in region $T_{1}$ (see Figure 3 ). The other cases can be proven similarly.

Let $V=P_{0}-A=(l \cos \theta, l \sin \theta)$ with $l=\left\|P_{0}-A\right\|$ and $\theta$ the angle between $A B$ and $V$. Now consider the curve $\mathbf{g}(t)$ from $A$ to $P_{0}$ on $\mathbf{Q}(u, v)$. Let $\mathbf{g}(t)=\mathbf{Q}(A+t V)$ and $d(t)=D(A+t V)=\|\mathbf{R}(A+t V)\|+k|w(A+t V)|$. Then $\mathbf{g}(0)=\mathbf{Q}(A)$ and $\mathbf{g}(1)=\mathbf{Q}\left(P_{0}\right)$. So $d(0)=D(A)=0$ and $d(1)=D\left(P_{0}\right)=\sup _{(u, v) \in T}\{D(u, v)\}$. Like the argument in Lemma 1, here we also consider three cases:

case 1. $w\left(P_{0}\right)=0 . \quad$ Applying (2) gives

$$
\begin{aligned}
D(u, v) & \leq d\left(P_{0}\right)=\left\|\mathbf{R}\left(P_{0}\right)\right\| \leq \frac{1}{8}\left(\sup \left\|\mathbf{R}_{u u}^{\prime \prime}\right\| \delta_{u}^{2}+2 \sup \left\|\mathbf{R}_{u v}^{\prime \prime}\right\| \delta_{u} \delta_{v}+\sup \left\|\mathbf{R}_{v v}^{\prime \prime}\right\| \delta_{v}^{2}\right) \\
& \leq \frac{1}{8}\left(D_{u u} \delta_{u}^{2}+2 D_{u v} \delta_{u} \delta_{v}+D_{v v} \delta_{v}^{2}\right)
\end{aligned}
$$




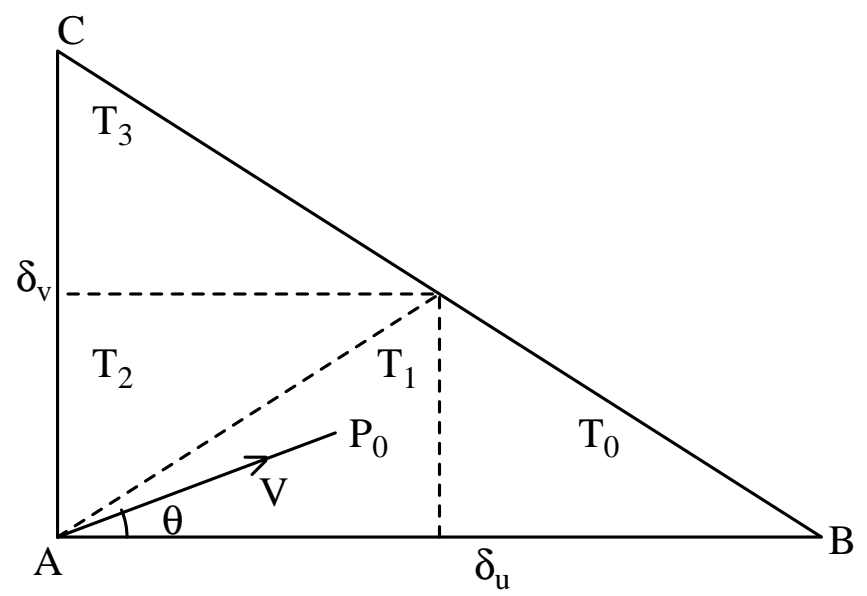

Fig. 3. The region of parametric triangle $T$.

case $2 . \mathbf{R}\left(P_{0}\right)=0 . \quad$ Just as in case 1 ,

$$
\begin{aligned}
D(u, v) & \leq d\left(P_{0}\right)=k\left|w\left(P_{0}\right)\right| \leq \frac{1}{8}\left(\sup \left|w_{u u}^{\prime \prime}\right| \delta_{u}^{2}+2 \sup \left|w_{u v}^{\prime \prime}\right| \delta_{u} \delta_{v}+\sup \left|w_{v v}^{\prime \prime}\right| \delta_{v}^{2}\right) \\
& \leq \frac{1}{8}\left(D_{u u} \delta_{u}^{2}+2 D_{u v} \delta_{u} \delta_{v}+D_{v v} \delta_{v}^{2}\right)
\end{aligned}
$$

case $3 . w\left(P_{0}\right) \neq 0, \mathbf{R}\left(P_{0}\right) \neq 0$.

Thus $d(t)$ is $C^{2}$ at $t=1$ and $d^{\prime}(1)=0$, i.e.

$$
d^{\prime}(1)=\frac{\mathbf{R}\left(P_{0}\right)}{\left\|\mathbf{R}\left(P_{0}\right)\right\|}\left(\mathbf{R}_{u}^{\prime}\left(P_{0}\right) l \cos \theta+\mathbf{R}_{v}^{\prime}\left(P_{0}\right) l \sin \theta\right)+k \frac{w\left(P_{0}\right)}{\left|w\left(P_{0}\right)\right|}\left(w_{u}^{\prime}\left(P_{0}\right) l \cos \theta+w_{v}^{\prime}\left(P_{0}\right) l \sin \theta\right)=0
$$

Writing $\mathbf{g}(t)$ in its Taylor expansion at $t=1$ :

$$
\mathbf{g}(t)=\mathbf{g}(1)+\mathbf{g}^{\prime}(1)(t-1)+\int_{1}^{t} \mathbf{g}^{\prime \prime}(s)(t-s) d s
$$

Letting $t=0$ gives

$$
\mathbf{g}(0)=\mathbf{g}(1)-\mathbf{g}^{\prime}(1)+\int_{0}^{1} \mathbf{g}^{\prime \prime}(s) s d s
$$

or

$$
0=\left(\mathbf{R}(A), w(A)=\left(\mathbf{R}\left(P_{0}\right), w\left(P_{0}\right)\right)-\left(\mathbf{R}_{u}^{\prime}\left(P_{0}\right), w_{u}^{\prime}\left(P_{0}\right)\right) l \cos \theta-\left(\mathbf{R}_{v}^{\prime}\left(P_{0}\right), w_{v}^{\prime}\left(P_{0}\right)\right) l \sin \theta+I\right.
$$

where

$$
I=\int_{0}^{1}\left(\left(\frac{\partial^{2} \mathbf{R}(s)}{\partial u^{2}}, \frac{\partial^{2} w(s)}{\partial u^{2}}\right) l^{2} \cos ^{2} \theta+2\left(\frac{\partial^{2} \mathbf{R}(s)}{\partial u \partial v}, \frac{\partial^{2} w(s)}{\partial u \partial v}\right) l^{2} \cos \theta \sin \theta+\left(\frac{\partial^{2} \mathbf{R}(s)}{\partial v^{2}}, \frac{\partial^{2} w(s)}{\partial v^{2}}\right) l^{2} \sin ^{2} \theta\right) s d s
$$

Taking the dot product of equation (30) with $\left(\frac{\mathbf{R}\left(P_{0}\right)}{\left\|\mathbf{R}\left(P_{0}\right)\right\|}, k \frac{w\left(P_{0}\right)}{\left|w\left(P_{0}\right)\right|}\right)$ yields

$$
0=\left\|\mathbf{R}\left(P_{0}\right)\right\|+k\left|w\left(P_{0}\right)\right|-0-0+I \cdot\left(\frac{\mathbf{R}\left(P_{0}\right)}{\left\|\mathbf{R}\left(P_{0}\right)\right\|}, k \frac{w\left(P_{0}\right)}{\left|w\left(P_{0}\right)\right|}\right)
$$

Therefore we get

$$
\begin{aligned}
d\left(P_{0}\right) & \leq\left|I \cdot\left(\frac{\mathbf{R}\left(P_{0}\right)}{\left\|\mathbf{R}\left(P_{0}\right)\right\|}, k \frac{w\left(P_{0}\right)}{\left|w\left(P_{0}\right)\right|}\right)\right| \\
& \leq \int_{0}^{1}\left[\left(\left\|\mathbf{R}_{u u}^{\prime \prime}(s)\right\|+k\left|w_{u u}^{\prime \prime}(s)\right|\right) \delta_{u}^{2} / 4+2\left(\left\|\mathbf{R}_{u v}^{\prime \prime}\right\|+k\left|w_{u v}^{\prime \prime}\right|\right) \delta_{u} \delta_{v} / 4+\left(\left\|\mathbf{R}_{v v}^{\prime \prime}\right\|+k\left|w_{v v}^{\prime \prime}\right|\right) \delta_{v}^{2} / 4\right] s d s \\
& \leq \frac{1}{4}\left(D_{u u} \delta_{u}^{2}+2 D_{u v} \delta_{u} \delta_{v}+D_{v v} \delta_{v}^{2}\right) \int_{0}^{1} s d s \leq \frac{1}{8}\left(D_{u u} \delta_{u}^{2}+2 D_{u v} \delta_{u} \delta_{v}+D_{v v} \delta_{v}^{2}\right)
\end{aligned}
$$


From Lemma 2 and Theorem 2, we can derive the required step sizes for rational surfaces:

THEOREM 4. Let $T \subset E^{2}$ be a right triangle with vertices $A, B$ and $C: B=A+\left(\delta_{u}, 0\right), C=A+\left(0, \delta_{v}\right)$. $\mathbf{r}(u, v)=\frac{\mathbf{R}(u, v)}{w(u, v)},(w(u, v)>0): T->E^{3}$ is a $C^{2}$ rational surface. $\mathbf{L}(u, v)$ is a fractional linearly parameterized triangle with $\mathbf{r}(A)=\mathbf{L}(A), \mathbf{r}(B)=\mathbf{L}(B)$ and $\mathbf{r}(C)=\mathbf{L}(C)$. Then $\sup _{(u, v) \in T}\|\mathbf{r}(u, v)-\mathbf{L}(u, v)\| \leq \epsilon$ if

$$
D_{u u} \delta_{u}^{2}+2 D_{u v} \delta_{u} \delta_{v}+D_{v v} \delta_{v}^{2} \leq 8 \epsilon \inf _{(u, v) \in T} w(u, v)
$$

where

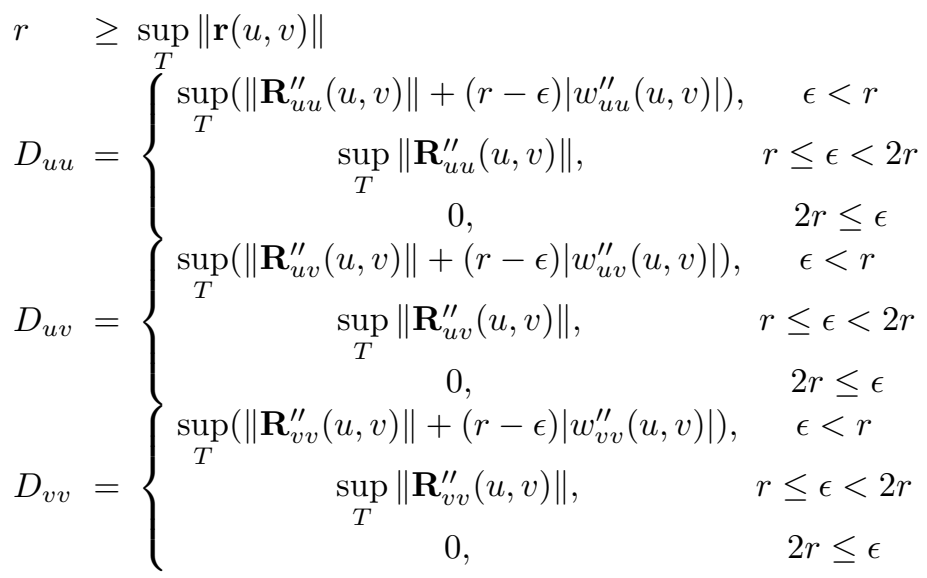

Note that formula (31) has two unknowns $\delta_{u}$ and $\delta_{v}$. It requires a second condition to determine them. In case $D_{u u}=0$ or $D_{v v}=0$, which implies the surface is fractional linear in the corresponding direction, we let $\delta_{u}=1$ and get

$$
\delta_{v}=\frac{\sqrt{D_{u v}^{2}+8 D_{v v} \epsilon \inf \{w(u, v)\}}-D_{u v}}{D_{v v}}
$$

or let $\delta_{v}=1$ and get

$$
\delta_{u}=\frac{\sqrt{D_{u v}^{2}+8 D_{u u} \epsilon \inf \{w(u, v)\}}-D_{u v}}{D_{u u}}
$$

Otherwise, set $\delta_{u} / \delta_{v}=\sqrt{D_{v v} / D_{u u}}$ and we have

$$
\delta_{u}=\sqrt{\frac{4 D_{v v} \epsilon \inf \{w(u, v)\}}{D_{u u} D_{v v}+D_{u v} \sqrt{D_{u u} D_{v v}}}}, \quad \delta_{v}=\sqrt{\frac{4 D_{u u} \epsilon \inf \{w(u, v)\}}{D_{u u} D_{v v}+D_{u v} \sqrt{D_{u u} D_{v v}}}}
$$

This setting will minimize the number of triangles for tessellating the surface, as indicated in [Abi-Ezzi and Wozny 1991]. A special case is $D_{u u}=D_{v v}=0$ and $D_{u v} \neq 0$. We simply let $\delta_{u}=\delta_{v}$, and thus

$$
\delta_{u}=\delta_{v}=\sqrt{\frac{4 \epsilon \inf \{w(u, v)\}}{D_{u v}}}
$$

For a rational Bézier surface expressed by

$$
\mathbf{r}(u, v)=\frac{\mathbf{R}(u, v)}{w(u, v)}=\frac{\sum_{i=0}^{n} \sum_{j=0}^{m} w_{i j} \mathbf{P}_{i j} B_{i}^{n}(u) B_{j}^{m}(v)}{\sum_{i=0}^{n} \sum_{j=0}^{m} w_{i j} B_{i}^{n}(u) B_{j}^{m}(v)}, u, v \in[0,1]
$$


$\mathbf{R}(u, v)$ and $w(u, v)$ are in Bézier form, and the evaluation of $D_{u u}, D_{u v}$ and $D_{v v}$ is easy.

$$
\begin{aligned}
D_{u u}=n(n-1) \max _{\substack{0 \leq i \leq n-2 \\
0 \leq j \leq m}}\left\{\left\|w_{i+2, j} \mathbf{P}_{i+2, j}-2 w_{i+1, j} \mathbf{P}_{i+1, j}+w_{i, j} \mathbf{P}_{i, j}\right\|+(r-\epsilon)\left|w_{i+2, j}-2 w_{i+1, j}+w_{i, j}\right|\right\} \\
\begin{aligned}
D_{u v}=n m \max _{\substack{0 \leq i \leq n-1 \\
0 \leq j \leq m-1}}\left\{\left\|w_{i+1, j+1} \mathbf{P}_{i+1, j+1}-w_{i+1, j} \mathbf{P}_{i+1, j}-w_{i, j+1} \mathbf{P}_{i, j+1}+w_{i, j} \mathbf{P}_{i, j}\right\|\right. \\
\left.+(r-\epsilon)\left|w_{i+1, j+1}-w_{i+1, j}-w_{i, j+1}+w_{i, j}\right|\right\} \\
D_{v v}=m(m-1) \max _{\substack{0 \leq j \leq m-2 \\
0 \leq i \leq n}}\left\{\left\|w_{i, j+2} \mathbf{P}_{i, j+2}-2 w_{i, j+1} \mathbf{P}_{i, j+1}+w_{i, j} \mathbf{P}_{i, j}\right\|+(r-\epsilon)\left|w_{i, j+2}-2 w_{i, j+1}+w_{i, j}\right|\right\}
\end{aligned}
\end{aligned}
$$

where $r$ can be chosen to be $r=\max _{\substack{0 \leq i \leq n \\ 0 \leq j \leq m}}\left\|\mathbf{P}_{i j}\right\|$.

It can be shown that the above estimation improves Cheng's result for surfaces. Also further improvements may be possible by bounding the second order derivatives with the "weight points", or by transforming the control points with a certain translation.

\section{CONCLUSION}

We have presented an approach to computing the tessellation step sizes for rational curves and surfaces. The method is derived in the homogeneous coordinates, so it is numerically accessible. The new formulas make a substantial improvement over previous known results. This is achieved by:

- efficiently estimating the effect of perspective transformation;

- treating $\left\|\mathbf{R}(t)-\mathbf{L}_{n}(t)\right\|+k\left|w(t)-L_{d}(t)\right|$ as a whole;

- performing more precise estimations.

Our approach is developed based on the parametric displacement of a curve or surface $\mathbf{r}$ from its fractional linearly parameterized interpolant L. Future work could consider the geometric displacement, i.e., the perpendicular distance of $\mathbf{r}$ from $\mathbf{L}$, for the global step size estimation. Reparameterization of the curve does not change the shape. In particular, the reparameterization by a Möbius transformation [Farouki 1997] might yield a larger step size. How to find an Möbius transformation that optimizes step size warrants further study.

\section{ACKNOWLEDGMENTS}

The authors are supported in part by NSF grant number CCR-9712407. Jianmin Zheng is also supported by Doctoral Science Foundation of China.

\section{REFERENCES}

Abi-EzzI, S. S. And Wozny, M. J. 1991. Factoring a homogeneous transformation for a more efficient graphics pipeline. Computers \& Graphics 15, 2, 249-258.

Cheng, F. April 1992. Estimating subdivision depths for rational curves and surfaces. ACM Transactions on Graphics 11, 2, 140-151. ISSN 0730-0301.

DE Boor, C. 1978. A Practical Guide to Splines. Springer.

ElBeR, G. 1996. Error bounded piecewise linear approximation of freeform surfaces. Computer-aided Design 28, 1, 51-57. FARIN, G. April 1993. Tighter convex hulls for rational Bézier curves. Computer Aided Geometric Design 10, 2, $123-126$.

Farouki, R. 1997. Optimal parameterizations. Computer Aided Geometric Design 14, 153-168.

Filip, D., Magedson, R., And Markot, R. 1986. Surface algorithms using bounds on derivatives. Computer Aided Geometric Design 3, 4, 295-311.

Floater, M. 1992. Derivatives of rational Bézier curves. Computer Aided Geometric Design 9, 3, 161-174.

Hermann, T. 1992. On a tolerance problem of parametric curves and surfaces. Computer Aided Geometric Design 9, 2, $109-118$.

KLASSEN, R. V. July 1994. Exact integer hybrid subdivision and forward differencing of cubics. ACM Transactions on Graphics 13, 3, 240-255. ISSN 0730-0301.

Lane, J. and Carpenter, L. November 1979. A generalized scan line algorithm for the computer display of parametrically defined surfaces. Computer Graphics and Image Processing 11, 290-297.

Lane, J. And Riesenfeld, R. 1980. A theoretical development for the computer generation and display of piecewise polynomial surfaces. IEEE Trans. Pattern Analysis Machine Intell. 2, 1, 35-46.

Piegl, L. A. And Richard, A. M. 1995. Tessellating trimmed nurbs surfaces. Computer-aided Design 27, 1, 16-26. 
RAPpoport, A. October 1991. Rendering curves and surfaces with hybrid subdivision and forward differencing. ACM Transactions on Graphics 10, 4, 323-341. ISSN 0730-0301.

Rockwood, A. P., Heaton, K., and Davis, T. July 1989. Real-time rendering of trimmed surfaces. Computer Graphics (Proceedings of SIGGRAPH 89) 23, 3, 107-116. Held in Boston, Massachusetts.

SchabACK, R. February 1993. Error estimates for approximations from control nets. Computer Aided Geometric Design 10, 1, $57-66$.

Sheng, X. And Hirsch, B. E. 1992. Triangulation of trimmed surfaces in parametric space. Computer-Aided Design 14, 437-444.

Tiller, W. 1992. Knot removal algorithms for nurbs curves and surfaces. Computer-Aided Design 24, 445-453.

Tookey, R. AND CRIPps, R. 1997. Improved surface bounds based on derivatives. Computer Aided Geometric Design 14, 8, 787-791. ISSN 0167-8396.

Wang, G.-J., Sederberg, T., And Saito, T. 1997. Partial derivatives of rational Bézier surfaces. Computer Aided Geometric Design 14, 4, 377-381.

WANG, G.-Z. 1984. The subdivision method for finding the intersection between two Bézier curves or surfaces. Zhejiang University Journal: Special Issue on Computational Geometry 14, 108-119. 